\title{
Comportement en flexion des poutres en matériaux sandwichs
}

\author{
Mustapha Assarar $^{1, a}$, Abderrahim El Mahi ${ }^{1}$, Mohammed Khawar Farooq ${ }^{2}$ \\ ET JEAN-MARIE BERTHELOT ${ }^{1}$ \\ 1 Groupe Composites et Structures Mécaniques, Université du Maine, Avenue Olivier Messiaen, 72085 Le Mans Cedex 9, France \\ ${ }^{2}$ Laboratoire d'Acoustique, Université du Maine, UMR CNRS 6613, Avenue Olivier Messiaen, 72085 Le Mans Cedex 9, France
}

Reçu le 20 mars 2004, accepté le 14 octobre 2004

\begin{abstract}
Résumé - Les matériaux sandwichs résultent généralement de l'association de deux semelles ou peaux de faible épaisseur, constituées de matériaux à haute résistance et haut module et d'une âme beaucoup plus épaisse et de faible densité. Ils ont pour avantages : une grande rigidité, une résistance à la rupture élevée associées à un faible poids par unité de surface. Malgré une activité de recherche et de développement sur ces matériaux, le comportement au cours de l'endommagement reste peu connu. L'objectif de cette étude est l'analyse du comportement en flexion 3-points de différents sandwichs au cours de la fatigue. Ils sont constitués de fibres de verre et de résine époxyde pour les peaux et de mousse PVC de différentes densités pour l'âme. Les essais de fatigue sont menés en contrôle déplacement. La réduction de la rigidité ou cours du cyclage est utilisée pour caractériser l'état d'endommagement des sandwichs. L'observation des faciès de rupture ont permis de relever les différents modes d'endommagement entraînant la rupture de l'éprouvette. Enfin, les durées de vie sont caractérisées par les courbes de Wöhler en utilisant le critère $N_{10}$, correspondant à une réduction de la rigidité de $10 \%$ par rapport à sa valeur initiale.
\end{abstract}

Mots clés : Matériaux sandwichs / flexion 3-points / fatigue / endommagement / durée de vie / courbes de Wöhler

\begin{abstract}
Behaviour of sandwich composites in flexural tests. Sandwich panels have a very high stiffness to weight ratio, which makes them particularly useful in the aerospace, marine and in almost in all the industrial applications. However, traditional sandwich structures present problems like their tendency towards delamination, interfacial debonding and other complicated modes of failures. The aim of the present study is to investigate the mechanical behaviour of sandwich composite materials under dynamic loading. They consist of epoxy resin and glass fibres for the skins and PVC foam of various densities for the core. These tests were carried out under displacement control at various displacement levels. Stiffness degradation is used to study the mechanical behaviour of sandwich composites in fatigue. The fracture topographies are observed by optical and scanning electronic microscopes. Results shows that the mode of damage and failure strongly depends upon the core densities. Finally fatigue life is characterised by Wohler curves using $N_{10}$ criterion for different types of sandwich composites.
\end{abstract}

Key words: Sandwich materials / 3-point bending / fatigue / damage / fatigue life / Wöhler curves

\section{Introduction}

Le comportement en fatigue des structures sandwichs joue un rôle très important quant à leur utilisation dans l'aéronautique et dans la marine. Cela est dû à la répercussion du mouvement des vagues sur les coques des bateaux et bien sûr à l'influence des vibrations des ailes des avions à la rencontre d'un trou d'air dans l'espace. C'est à partir de cela que les études de fatigue sur

\footnotetext{
a Auteur correspondant :

mustapha.assarar .etu@univ-lemans.fr
}

les plaques sandwichs ont commencé. Les résultats de ces études évoluent en fonction de l'évolution des matériaux composites.

Dans le cas d'une structure sollicitée en flexion, la rigidité et la résistance en flexion augmentent rapidement avec l'épaisseur des stratifiés constituant la structure. Toutefois, pratiquement seules les couches externes supportent les charges imposées à la structure et un accroissement des propriétés spécifiques en flexion est obtenu en remplaçant les couches internes par un matériau léger et en l'interposant entre les couches externes pour obtenir 
un matériau sandwich. Un tel matériau composite est donc constitué de deux peaux qui supportent l'essentiel du chargement mécanique imposé et d'une âme dont la fonction est de transmettre par cisaillement le chargement mécanique entre les deux peaux. Ce type de matériaux qui associés de bonnes propriétés mécaniques et acoustiques à un coût relativement faible est bien adapté aux applications industrielles de grandes diffusions. La connaissance des propriétés statiques ne suffit pas, le développement de leur emploi nécessite une information complémentaire sur leurs propriétés en fatigue et leur durabilité.

L'objectif de notre travail est d'évaluer le comportement en fatigue des matériaux sandwichs constitués d'une âme en mousse expansée et de peaux stratifiées en fibres de verre et de résine époxyde.

\section{Matériaux et essais}

Différents types de sandwichs sont élaborés au laboratoire (Groupe Composites et Structures Mécaniques). Ils sont constitués de stratifiés à fibres de verre et résine époxyde de type $[0 / 90]_{\mathrm{s}}$ pour les peaux (1 mm d'épaisseur) et de mousse en PVC de différentes densités $\left(60 \mathrm{~kg} \cdot \mathrm{m}^{-3}, 80 \mathrm{~kg} \cdot \mathrm{m}^{-3}, 100 \mathrm{~kg} . \mathrm{m}^{-3}\right.$ et $200 \mathrm{~kg} . \mathrm{m}^{-3}$ ) pour l'âme (15 mm d'épaisseur). Les éprouvettes ont une longueur $L$ de $300 \mathrm{~mm}$, une largeur $b$ de $50 \mathrm{~mm}$ et une épaisseur $h$ égale à l'épaisseur de la plaque qui est de $17 \mathrm{~mm}$. La distance $l$ entre appuis est de $250 \mathrm{~mm}$.

Les éprouvettes sont sollicitées en flexion 3-points sur une machine hydraulique universelle type INSTRON, munie d'une cellule de charge de $5 \mathrm{kN}$ pour la mesure de la force et d'un capteur LVDT pour la mesure du déplacement. Ces essais sont menés en contrôle déplacement, le déplacement moyen et maintenu constant égal à $50 \%$ du déplacement à la rupture en statique. La forme d'onde est sinusoïdale d'une fréquence de $10 \mathrm{~Hz}$. Plusieurs niveaux de chargement $r\left(r=d_{\text {max }} / d_{\text {rup }}\right)$ sont considérés pour tracer les courbes de perte de rigidité et pour construire les diagrammes d'endurances (courbes de Wöhler), où $d_{\max }$ est le déplacement maximum appliqué en fatigue et $d_{\text {rup }}$ est le déplacement à rupture en statique.

\section{Résultat et discussion}

La figure 1 donne un résultat typique des essais de fatigue. Elle représente l'évolution de la contrainte maximale en fonction du nombre de cycles en fatigue, la contrainte maximale $\sigma_{\max }$ est rapportée à celle obtenue dans le premier cycle $\sigma_{0 \max }$. La diminution de la contrainte jusqu'à la rupture des éprouvettes se déroule en trois phases : dans un premier temps se manifeste une diminution brutale dès les premiers cycles; la diminution devient ensuite très lente dans la deuxième phase, correspondant à la quasi-totalité de la durée de vie de l'éprouvette, enfin dans la troisième phase qui est très courte où la perte de charge s'accélère brutalement jusqu'à la rupture de l'éprouvette. Les trois phases de la

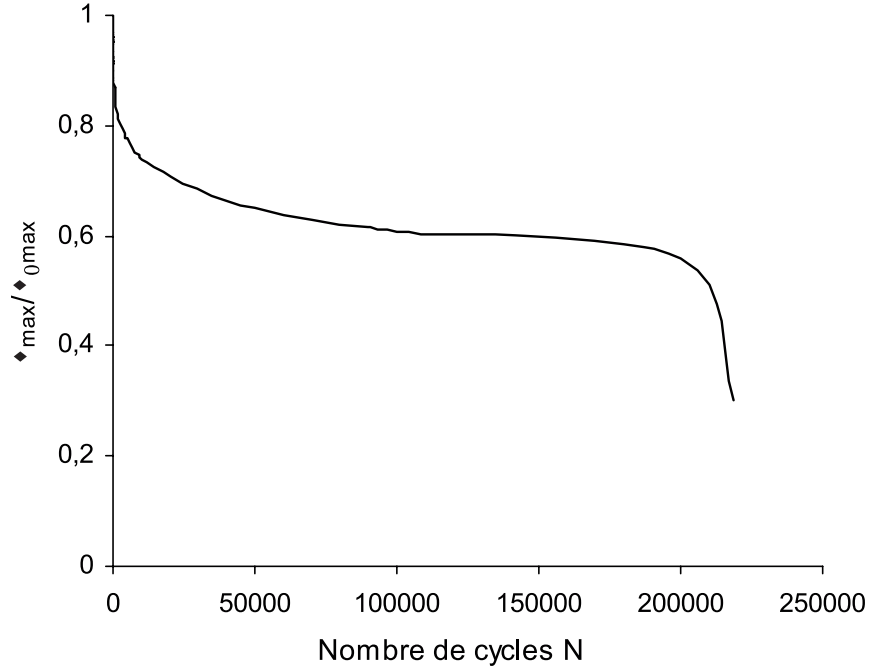

Fig. 1. Évolution de la contrainte en fonction du nombre de cycles.

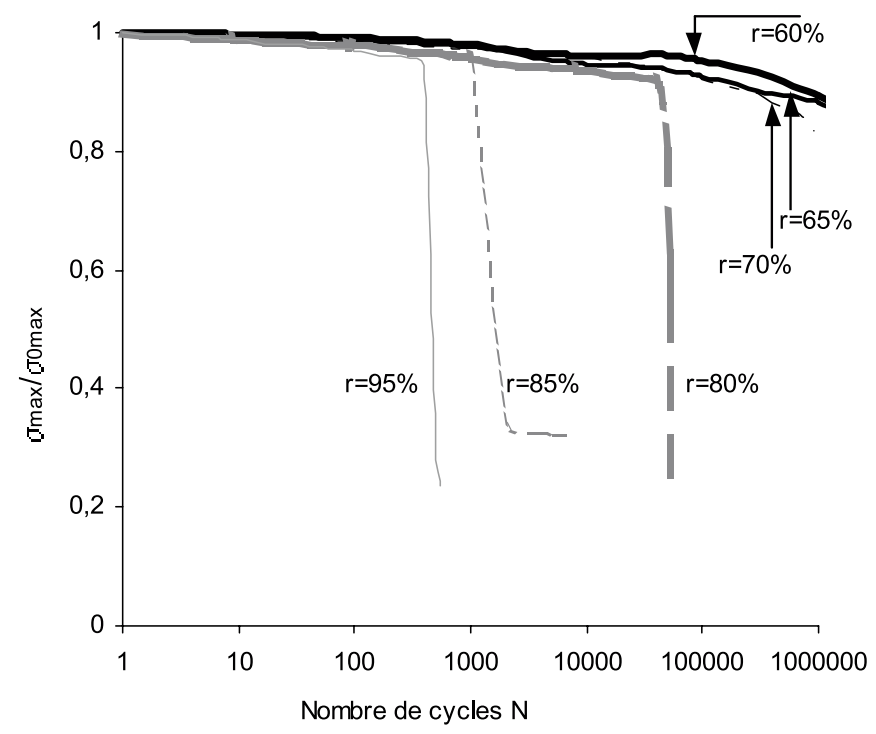

Fig. 2. Évolution de la contrainte $\left(\sigma_{\max } / \sigma_{0 \max }\right)$ en fonction du nombre de cycles pour différents niveaux de chargement dans le sandwich $\left(200 \mathrm{~kg} \cdot \mathrm{m}^{-3}\right)$.

courbe peuvent être associées à la multiplication de la fissuration dans la résine dans les peaux pour la première phase, l'initiation et la propagation de la décohésion entre la peau en contact avec l'appui central et l'âme durant la deuxième phase et enfin à la rupture des fibres entraînent la rupture de la peau supérieure pour la dernière phase.

Nous avons reporté sur la figure 2 avec une échelle semi-logarithmique, l'évolution de la perte de rigidité $\left(\sigma_{\max } / \sigma_{0 \max }\right)$ en fonction du nombre de cycles pour différents niveaux de chargement $r\left(d_{\max } / d_{\text {rup }}\right)$ dans la cas du sandwich constitue par la mousse la plus dense $\left(200 \mathrm{~kg} . \mathrm{m}^{-3}\right)$. Nous constatons bien évidemment que la durée de vie augmente avec la diminution de $r$. Pour un niveau de chargement $r$ faible, la rupture n'est pas atteinte même lorsque le nombre de cycles est supérieur 


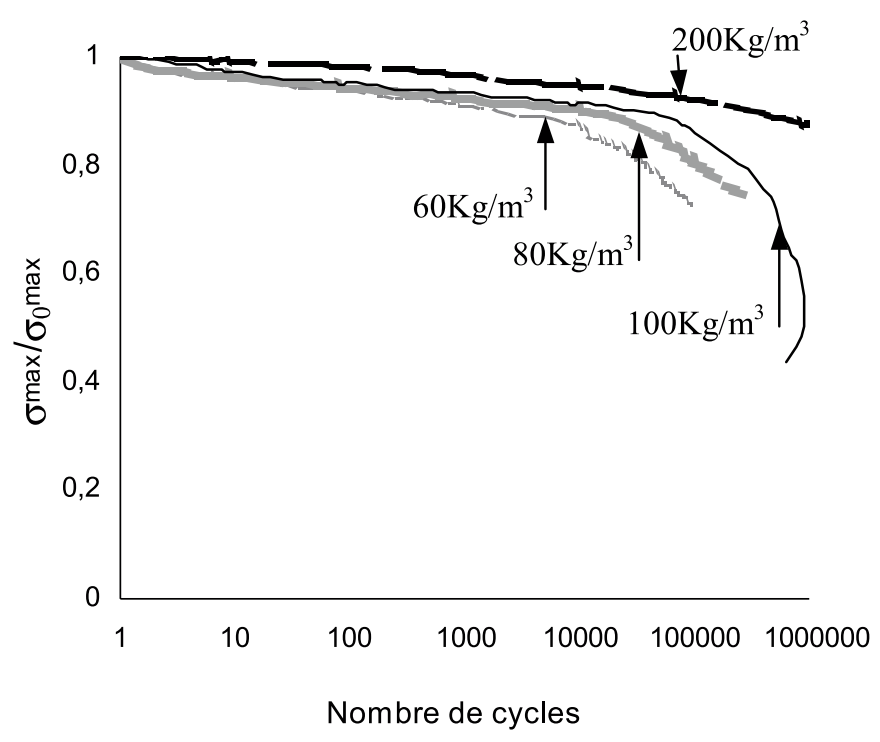

Fig. 3. Évolution de la contrainte $\left(\sigma_{\max } / \sigma_{0 \max }\right)$ en fonction du nombre de cycles dans différents sandwichs pour un niveau de chargement $r=80 \%$.

à $10^{6}$, alors que pour des valeurs élevées de $r$ la rupture est observée au bout de quelques centaines de cycles.

L'analyse des résultats montre que le comportement jusqu'à la rupture sous sollicitations cycliques dépend à la fois du rapport de chargement et de la nature de l'âme. Le déplacement moyen détermine les mécanismes d'endommagement activés au début du cyclage, alors que l'amplitude détermine la vitesse de propagation et de multiplication de ces mécanismes. Pour un déplacement moyen contant égal $50 \%$ de la valeur à la rupture en statique on constate que :

- Lorsque le niveau de chargement est faible correspondant à une faible amplitude, la propagation d'endommagement se fait très lentement, la durée de vie est ainsi élevée.

- Lorsque le niveau de chargement est important correspondant à une grande amplitude, la vitesse de croissance d'endommagement est très importante correspondant à une durée de vie très faible.

Pour mettre en évidence l'effet de la densité de l'âme du sandwich sur le comportement en fatigue, nous avons comparé pour un même niveau de chargement les résultats obtenus dans les quatre sandwichs étudiés. La figure 3 représente l'évolution de la contrainte en fonction du nombre de cycles pour un niveau de chargement égal à $80 \%$. La première phase des courbes charge-nombre de cycles semble similaire dans les quatre sandwichs, elle corresponde à la dégradation de la résine dans les couches à $90^{\circ}$ des peaux du sandwich. La deuxième phase correspond à l'initiation de la fissure âme-peau autour de l'appui central et sa propagation à l'interface. Cette fissure est initiée assez rapidement dans le sandwich avec une âme de faible densité $\left(60 \mathrm{~kg} . \mathrm{m}^{-3}\right)$, et sa propagation se fait rapidement. Les sandwichs constitués d'âmes de densités élevées $\left(200 \mathrm{~kg} . \mathrm{m}^{-3}\right)$ possèdent donc une bonne résistance à la fatigue en flexion trois-points.

\section{Observation des faciès de rupture}

L'observation des faciès de rupture (Figs. 4 et 5) des différents échantillons testés au cours du cyclage, montre que la ruine de l'éprouvette est due à la rupture totale de la peau au contact avec l'appui central. Pour un faible niveau de chargement (Fig. 4) la croissance de l'endommagement en fonction du nombre de cycles au cours de la fatigue est gouvernée par la croissance de la décohésion entre la peau et l'âme, amorcée autour de l'appui central et entraînant la rupture des fibres et donc la rupture totale de la peau en contact de l'appui centrale. Par contre pour un niveau de chargement élevé (Fig. 5), l'endommagement se manifeste par une décohésion entre l'âme et la peau supérieure proche de l'appui central ensuite par des propagations des fissurations par cisaillement dans l'âme ce qui entraîne une autre décohésion entre l'âme et la peau inférieure entraînent la ruine finale du sandwich.

\section{Durée de vie}

Pour déterminer les performances des matériaux en fatigue, différents critères de la durée de vie $\left(N_{\mathrm{s}}, N_{3}, N_{5}\right.$, $N_{10}$ et $N_{\mathrm{R}}$ ) sont considérés dans la littérature à partir des courbes donnant l'évolution de la charge en fonction du nombre de cycles. Le critère le plus sévère est celui qui caractérise par la valeur $N_{\mathrm{s}}$ qui correspond au nombre de cycles à la fin du domaine linéaire. Les critères $N_{3}, N_{5}$ et $N_{10}$ correspondent respectivement à des chutes de $3 \%$, $5 \%$ et $10 \%$ de la rigidité par rapport à sa valeur initiale. Le critère $N_{\mathrm{R}}$ correspond au nombre de cycles à la rupture complète de l'éprouvette lorsqu'elle est atteinte. Pour notre étude, nous avons choisi le critère $N_{10}$ qui est le plus utilisé dans la littérature. Les durées de vie sont données dans les diagrammes d'endurances (Fig. 5). Ces diagrammes donnent l'évolution du niveau de chargement $(r)$ en fonction du nombre de cycles $N_{10}$, dans le cas d'un déplacement moyen maintenu constant égal à $50 \%$ du déplacement à la rupture en statique. L'analyse des résultats obtenus, montre une grande dispersion de la durée de vie. Cependant, il ressort que l'ensemble des résultats converge vers l'unité pour des faible nombre de cycles; ce qui montre que lorsque le niveau de chargement est voisin de celui de la rupture en statique, la rupture est obtenue dès les premiers cycles, par contre pour des faibles niveaux de chargement, la rupture est obtenue pour des nombres de cycles élevés.

Pour mieux prendre en compte l'effet de la densité de l'âme sur la durée de vie du sandwich, nous avons considéré une expression du type :

$$
r=A-B \log \left(N_{10}\right)
$$

Cette forme de description permet de mieux visualiser la tenue en fatigue des matériaux par l'intermédiaire de la valeur intrinsèque $B$, représentant la pente de la droite d'endurance. Cette valeur correspond au taux de chute du niveau de chargement admissible exprimé en $\%$ décade par cycle. La valeur de $B$ du sandwich constitué 


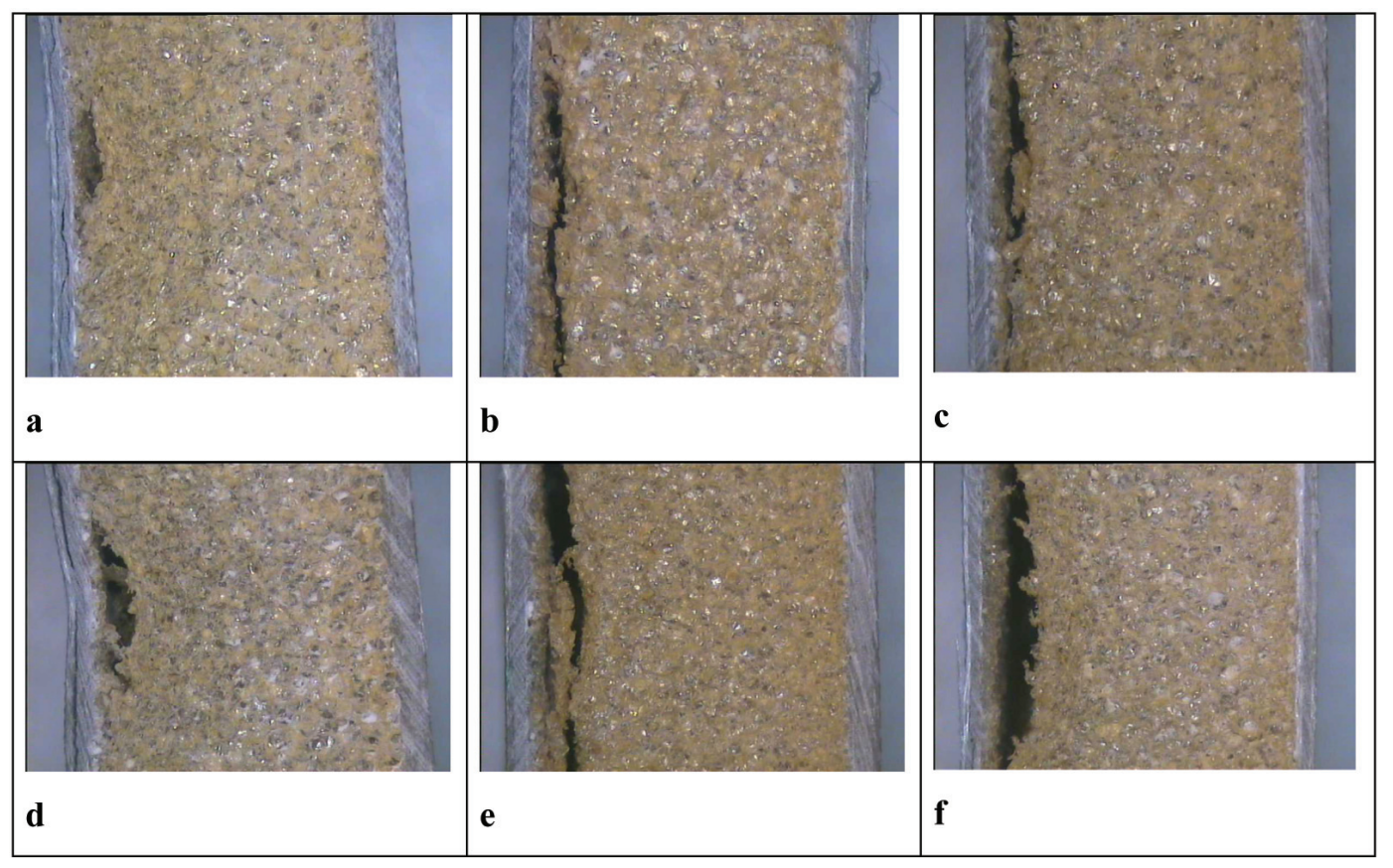

Fig. 4. Propagation de l'endommagement en fonction du nombre de cycles.

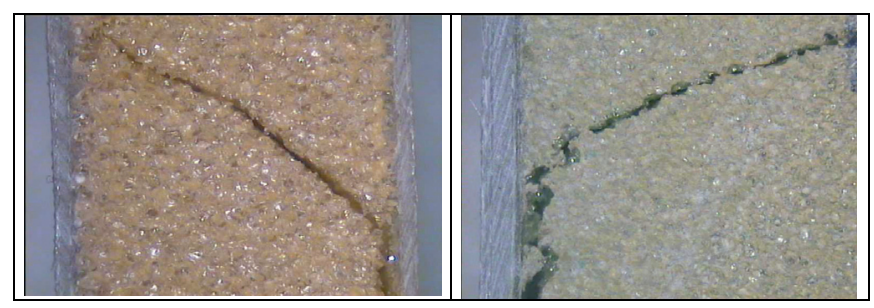

Fig. 5. Rupture par cisaillement.

par la mousse la plus dense est faible comparativement aux autres sandwichs. Cela signifie, que pour un même niveau de chargement la durée de vie augmente lorsque la densité de la mousse augmente.

Les essais d'endurance se caractérisent par une dispersion des valeurs de la durée de vie dépendant de plusieurs paramètres tels que la nature de peau de la densité et l'épaisseur de l'âme, etc. D'une manière générale, cette dispersion est principalement liée à la nature hétérogène des sandwichs. En outre, les éprouvettes n'ont pas toujours des caractéristiques comparables : fraction volumique, distribution des défauts, résistance à la rupture en statique, etc. La rupture en fatigue dépend d'une série de processus aléatoires dont la conjugaison se traduit par une dispersion dans les résultats de durée de vie entre les éprouvettes soumises à un même niveau de chargement $r$ et sollicitées dans les mêmes conditions.

\section{Conclusion}

L'objectif de ce travail était d'analyser le comportement des matériaux sandwichs en fatigue. Les premières analyses d'endommagement qui ont été observées ont

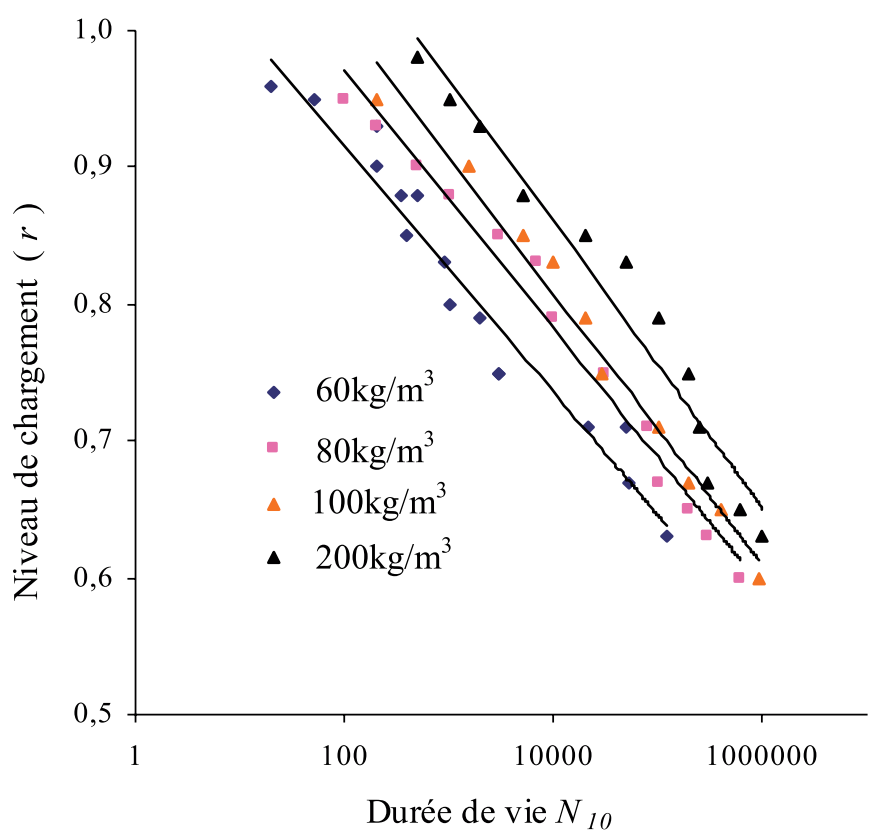

Fig. 6. Durée de vie en fatigue.

montré l'importance du phénomène de décohésion entre la peau et l'âme au voisinage de l'appui central entraînant la rupture du sandwich. L'influence de la densité de l'âme sur la résistance à la fatigue des matériaux sandwichs a été mise en évidence par l'établissement d'un critère de durée de vie à partir des courbes d'endurances. Les résultats obtenus ont montré que la densité de l'âme joue un rôle important sur la durée de vie de ces matériaux. 


\section{Références}

[1] H.T. Hahn, L. Lorenzo, Fatigue failure mechanisms in composite laminates, Advances in Fracture Research, 6th International conference fracture, New Delhi, India, 4-10 Dec. 1984, pp. 549-568

[2] G. Caprino, A. D'Amore, Flexural fatigue behaviour of random continuous fibre reinforced thermoplastic composites, Composite Science and Technology, 58 (1998) 957-965

[3] P. Fournier, O. Pelissou, A. Chateauminois, B. Large-Toumi, Post-buckling method applied to static and fatigue characterization of composites materials,
EACM Amsterdam, Ed. BCS, EACM-CTS, 1992, pp. 235-244

[4] J.F. Mandell, Fatigue behaviour of fibre resin composites, Developments in Reinforced Plastics, App. Sci. Pub., P.G. Pinchard (ed.) London-New York, 67, 1982

[5] M.-K. Farooq, A. El Mahi, S. Sahraoui, Flexural behaviour of sandwich composite materials under fatigue matériaux 2002, tours, octobre 2002

[6] A. El Mahi, M.-K. Farooq, S. Sahraoui, A. Bezasi, Modelling the flexural behaviour of sandwich composite materials under cyclic fatigue, Materials and design (2004) 199-208 